\title{
ASSESSMENT OF A KEYPOINTS DETECTOR FOR THE REGISTRATION OF INDOOR AND OUTDOOR HERITAGE POINT CLOUDS
}

\author{
Rami Assi, Tania Landes, Arnadi Murtiyoso, Pierre Grussenmeyer \\ ICube Laboratory UMR 7357, Photogrammetry and Geomatics Group, National Institute of Applied Sciences (INSA), \\ 24 Boulevard de la Victoire, 67084 Strasbourg Cedex, France \\ rami.assi@insa-strasbourg.fr; tania.landes@insa-strasbourg.fr; arnadi.murtiyoso@insa-strasbourg.fr; \\ pierre.grussenmeyer@insa-strasbourg.fr
}

\section{Commission II, WG II/8}

KEY WORDS: Indoor point clouds, Building information model, Descriptor, Segmentation, Registration

\begin{abstract}
:
In the context of architectural heritage preservation, constructing building information models is an important task. However, conceiving a pertinent model is a difficult, time consuming and user-dependent task. Our laboratory has been researching methods to decrease the time and errors inferred by manual segmentation of point clouds. In the perspective of automatization of the process, we implemented an automated registration method that used only keypoints. Keypoints are special points that hold more information about the global structure of the cloud. In order to detect keypoints, we used the Point Cloud Library (PCL) toolbox. The pertinence of the method was evaluated by registering more than 300 clouds of the zoological museum of Strasbourg. The quality of the keypoint detection was first verified on geo-referenced indoor point clouds. Then we applied this method to register the indoor and outdoor point clouds that have much less area in common; those common points being generally the doors and windows of the façade. The registrations of indoor point clouds were satisfying, with mean distances to the ground truth inferior to $20 \mathrm{~cm}$. While the first result for joint indoor/outdoor registration are promising, it may be improved in future works.
\end{abstract}

\section{INTRODUCTION}

The construction of building information model for heritage building documentation and maintenance is an active research field. A BIM is a virtual three-dimensional (3D) representation of a future or existing building including a geometrical model but also any pertinent semantic information related to structural elements or for the management of the building. Heritage buildings are older than the BIM technology. In order to exploit the advantages of a BIM, it must be created based on the existing building. A raw point cloud representation of the building is acquired on the field. Then the points are semi-automatically processed to produce the 3D model. The scan-to-BIM process often requires manual intervention that is time consuming, subject to errors and user dependent. Our work aims at the further automatization of the process. The laboratory's previous research in the field of BIM focused on semi-automatic methods for indoor segmentation (Macher et al., 2017) and outdoor segmentation (Boulaassal et al., 2010). To have a complete visualisation of the building, the indoor and outdoor point clouds must be registered. This is especially true when the outdoor data are produced in another method or at different dates than the indoor data. This is the case when mobile mapping systems are used to acquire all façades of a street and indoor data are acquired with static or mobile handheld systems. Therefore, indoor and outdoor datasets are not automatically registered together. In the absence of (precise) geo-referencing, we do not want to infer errors due to manual registration. Hence, outdoor and indoor point clouds automated registration is also an active research question.

The first part of our paper will sum up a few of our laboratory's previous studies and how they interact with each other as pieces of a larger pipeline. Researches have been conducted for indoor segmentation in floors and rooms, as well as outdoor façade segmentation. In these works, the segmentation process is mostly automated.

The second part describes our implementation of the Point Cloud Library (PCL) as proposed by Rusu and Cousins (2011) for the detection of keypoints in our datasets. Then we attempt to use the minimal information contained in those keypoints to register indoor point clouds. Furthermore, we assessed the efficiency of this method for the registration of indoor and outdoor point clouds.

The third and final section of the paper discusses the results obtained by studying keypoints features obtained on our data set. Algorithms were tested on data acquired in and around the zoological museum of Strasbourg (France) (Figure 1).

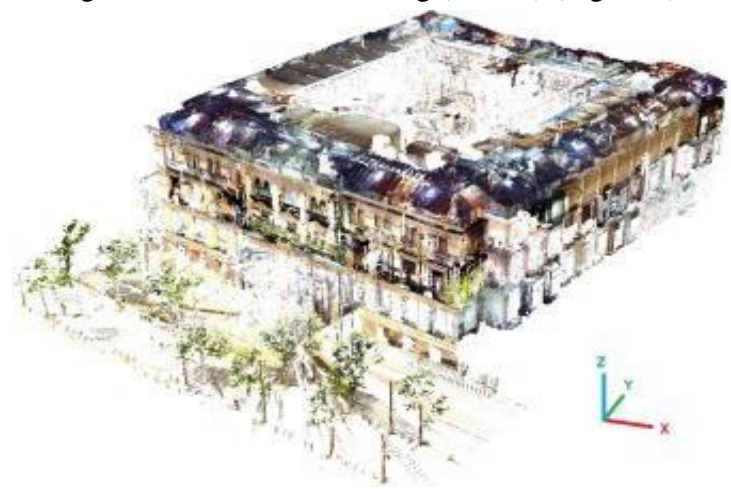

Figure 1. Complete indoor and partial outdoor point cloud of the zoological museum of Strasbourg.

Indoor data were acquired with static laser scanning technique and outdoor data with a mobile mapping system (Stereopolis from IGN). The museum consists of four floors and a vast attic, with 
an internal courtyard. The ground floor is composed of classrooms and laboratories. Two floors are dedicated to the visiting tours and are filled with life-sized animal models as well as complete skeletons. The third floor houses the employees' offices. Pieces of furniture obstruct the walls of most rooms. In the attic, old exposition models are stored. All these obstacles that cannot be removed during data acquisition make this dataset all the more pertinent in order to ascertain the robustness of the method.

\section{STATE-OF-THE-ART}

\subsection{Previous work}

Among our group's previous work are two segmentation pipelines that fit in the joint indoor, outdoor segmentation problem. The first is a façade segmentation algorithm (Boulaassal et al., 2010). It used the random sample consensus algorithm of RANSAC (Fischler and Bolles, 1981) to extract the largest planes of the façades. Then Delaunay triangulation of points belonging to façade's planar segments is computed. An interesting property of this triangulation method is that the edges of the resulting triangles are longer when connecting points on the edges or holes of the cloud. By isolating those longer segments and their corresponding points, we are able to highlight the points surrounding the holes of the façade i.e. windows and doors.

The second method is complementary as it focuses on automated indoor point cloud segmentation (Macher et al., 2017). The algorithm simplified the segmentation task by going through several subdivision of the building before the final segmentation. The study of the altitude histogram of the point cloud lets us determine the point belonging to the floor and to the ceiling on each floor. Then a region growing algorithm is applied on a horizontal slice to detect walls and separate each floor in rooms. Finally, a variant of RANSAC, i.e. MLESAC (Torr et al., 2000) is applied to segment each wall as planes.

As an extension of this pipeline, a first approach to opening detection in indoor point clouds was developed (Assi et al., 2019). For each segmented wall, all points belonging to it are projected on the corresponding 2D plane to form a binary image. Any openings or obstacles will be represented by white pixels in the black image. Image processing was then performed to clean the image of any remaining noise. Then the region growing implementation of MATLAB is applied to identify each white region. An energy function inspired by (Boykov et al., 2001/Wenzhong et al., 2019) is applied to differentiate regions resulting from the obstructions and actual openings. The function first eliminates regions that cannot coherently correspond to windows or doors. Then the function analysed the mean repartition of pixels in the bounding box of the region. More rectangular windows and doors will fill almost all pixels of the bounding box, resulting in a higher mean repartition. With the correct thresholds, the energy functions will discard wall obstructions and segment actual openings such as windows and doors.

\subsection{Keypoints detection}

In a point cloud, information can be redundant. Not all available properties are pertinent. It is all the more important in the context of point cloud segmentation. Let us consider a rectangular wall. Using laser scanning technique for the acquisition of a point cloud of this wall will result in thousands of points. For many processes such as segmentation, most of those points are superfluous. In the case of segmentation, the final resulting wall can be modelled with only 4 points: one for each corner of the wall. The main plane can be extracted with an algorithm such as RANSAC. Then the four points delimiting the wall can be found by looking for the extrema of distances relative to the centroid of the wall. This specific example would give good results with only hundreds or even tens of points. To process thousands of points is a waste of time. However, one cannot randomly pick a few points either. If we consider a sub cloud of the wall that do not include its edges, the resulting segmentation will be a smaller wall. On the other hand, the result will not change if we discard points within the wall. It means that not all points hold the same value of information. Some are more important than others: those are called keypoints. Keypoints' strength is to most of the pertinent information of the point cloud within a small percentage of it points. We will see in the study how "small" this percentage actually is.

The keypoints detection algorithm was chosen based on previous research (Mittet et al., 2014). Four keypoints detection methods were evaluated and compared for real time detection, i.e. SURF, SIFT, ORB, and GFTT. In their study the SIFT algorithm was discarded as it was too slow for real time detection. However, it has better stability to rotations and translations. As speed is not the current focus of our research, we chose PCL's SIFTKeypoint algorithm for its stability. Indeed, as described in the method, rigid transformations will be applied to our data.

Indoor/outdoor registration using SIFTKeypoint was previously considered in Murtiyoso et al. (2018). In this work, windows are manually segmented in the indoor and outdoor point clouds. Then keypoints are detected for both sides of the windows, resulting in two sets of keypoints.

\section{METHOD}

In order to acquire the complete point cloud of a building, we successively perform laser scanning in each room and outside the building. From each acquisition a different point cloud is computed. The complete point cloud of the building is obtained by combining all those point clouds like a puzzle (see Figure 2). This process is called registration.
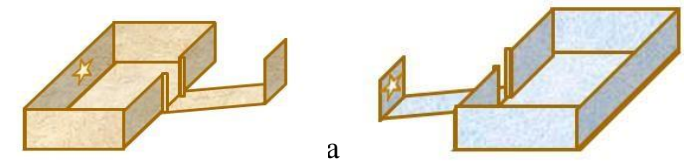

b

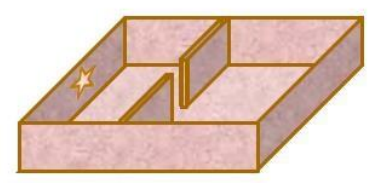

Figure 2. a - point cloud of a room including a target, the next room is partially acquired through the door $b$ - point cloud of a second room, the previous room is partially acquired through the door and includes the target $\mathrm{c}$ - using the targets as a common point, both point clouds are registered 
In order to precisely register point clouds, artificial targets may be necessary. The target is an object easily detectable for the laser scanning that is placed at the intersection of two-point clouds. It is detected in both point clouds and can be used to register them. A least 3 targets should be visible from two successive stations. Once they are detected in both point clouds, registration can be performed. As show in Figure 2 the stars represent overlapping areas of both clouds that may be used as targets for registration.

This process allows a quick registration of all indoor point clouds. However, it has limitations with outdoor point clouds. Indeed, there are no common points between indoor and outdoor where a target could be placed. That is why we investigate windows as a possible substitute (Figure 3 ). If we could detect windows in both indoor and outdoor point clouds, they could be used as targets for further registration.
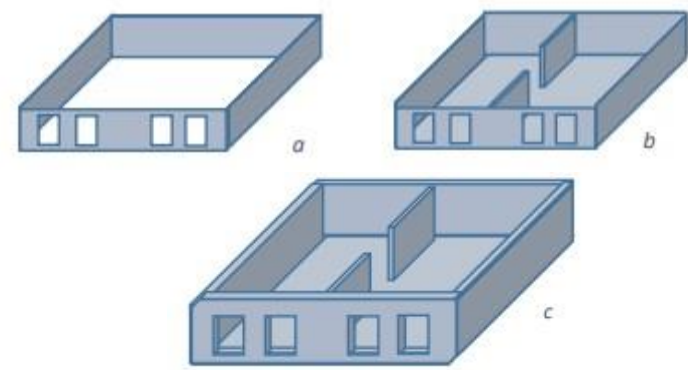

Figure 3. $\mathrm{a}$ - outdoor point cloud with detected openings; $\mathrm{b}$ indoor point cloud with detected openings; c - complete registered point cloud using windows as common points

In this study, we focus on the detection of keypoints using the PCL (Rusu et al., 2011) function SIFTKeypoint (Lowe, 2004). As windows are visible in indoor and outdoor point clouds, we expected good registration based on keypoints belonging to the windows. A general workflow of the developed algorithm can be seen in Figure 4. Each step is described in more details in the following section.

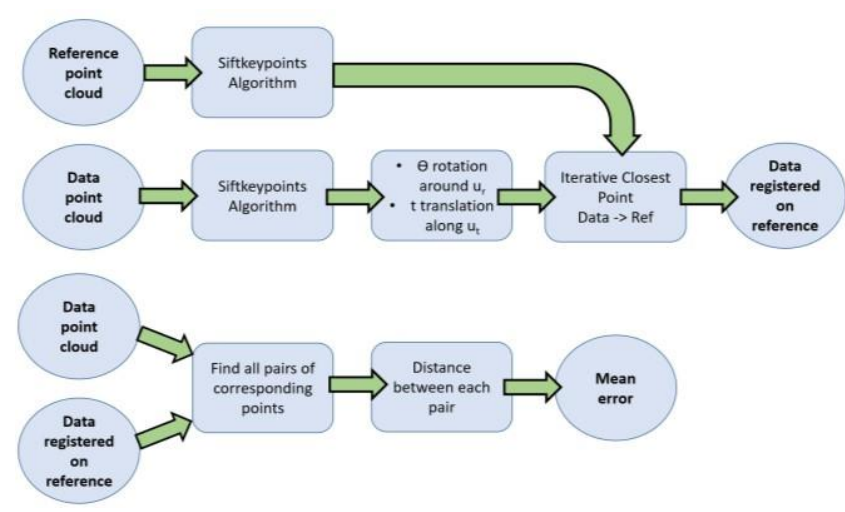

Figure 4: pipeline of the experiment.

- $0<\Theta<\pi / 4$ random

- $\left|\mathrm{u}_{\mathrm{r}}\right|=1$ random direction

- $0<\mathrm{t}<1$ meters random

- $\left|\mathrm{u}_{\mathrm{t}}\right|=1$ random direction

We consider the point clouds of the zoological museum of Strasbourg as the ground truth since they were already registered and georeferenced by conventional technique (measurements of targets by tacheometry) (Figure 1). The point clouds' files are roughly ordered so that 2 successive point clouds belong to actually connected rooms and may be registered.

For each pair of consecutive point clouds (Figure 5) we define a reference point cloud and a data point cloud that will be registered using the SIFTKeypoint algorithm. Keypoints are extracted for both the reference and data point clouds (Figure 6).

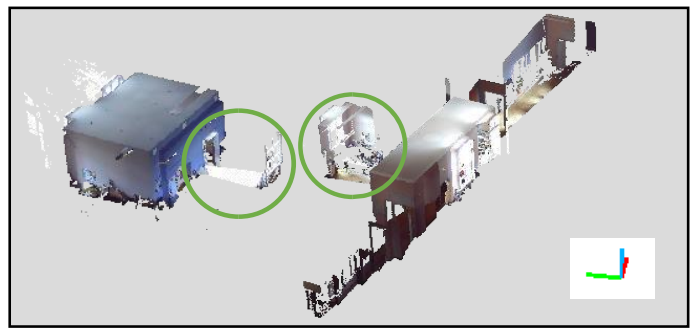

Figure 5. Two consecutive point clouds of a room (left) and a hallway (right). In each point cloud, points of the other one were acquired by lasers going through the door (green circles). Belonging to both clouds, those point will matter in the registration.

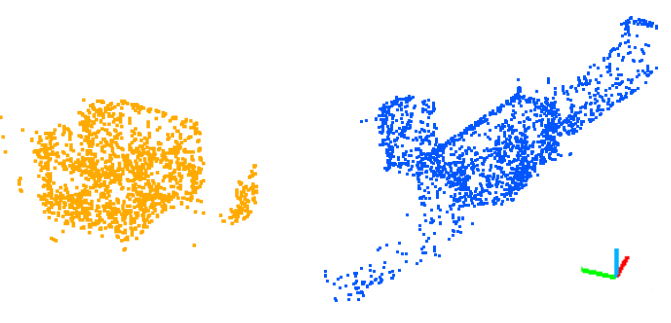

Figure 6. Keypoints extracted from the point clouds in Figure 4

As mentioned previously, our dataset is already registered so we need to "unregister" it by moving the data point cloud a bit. In order not to create a bias in this transformation, we applied a randomly generated transformation for each pair of consecutive point clouds (Figure 7).

We wanted the random transformation to make the data point cloud seems like it was roughly registered on the reference point cloud. So we applied a small rotation and small translation.

We randomly generate $M$, the $\Theta$ around the vector $u_{r}$ and $t$ translation along the vector $\mathrm{u}_{\mathrm{t} .}$ Both vectors are normalised. As we want the clouds to be roughly registered still, $\Theta$ and $t$ maximum possible values are respectively $\pi / 4$ and 2 meters.

$\mathrm{M}=$

$$
\left[\begin{array}{llll}
u_{x}^{2}(1-c)+1 & u_{x} u_{y}(1-c)-u_{z} s & u_{x} u_{z}(1-c)+u_{y} s & t_{x} \\
u_{x} u_{y}(1-c)+u_{z} s & u_{y}{ }^{2}(1-c)+1 & u_{y} u_{z}(1-c)-u_{x} s & t_{y} \\
u_{x} u_{z}(1-c)-u_{y} s & u_{y} u_{z}(1-c)+u_{x} s & u_{z}^{2}(1-c)+1 & t_{z} \\
0 & 0 & 0 & 1
\end{array}\right]
$$

The expression of $\mathrm{M}$ is given by (1). The rotation is applied by considering the center of the data point cloud as the origin. 

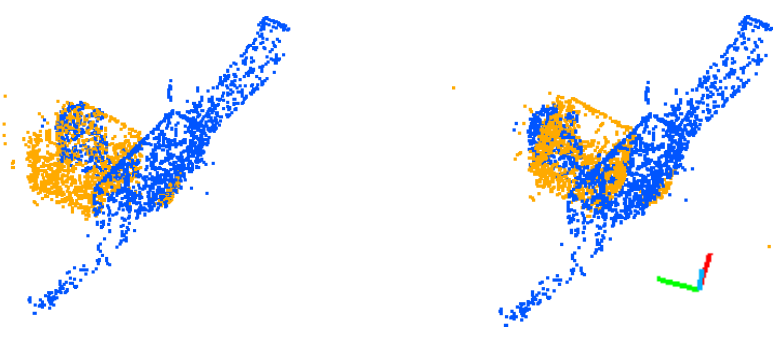

Figure 7. On the left the keypoints directly after extraction from the registered point clouds (ground truth). On the right, the reference cloud (blue) has not changed, but the data cloud (orange) was randomly transformed.

At this point we have the reference point cloud's keypoints and the data point cloud's keypoints which are unregistered. We use CloudCompare's implementation of iterative closest point (ICP) to register the data with the reference point cloud. In theory, the transformation computed by ICP should be $\mathrm{M}^{-1}$.

We evaluated the error of registration by measuring the mean distance of points between the registered data keypoints and the original data keypoints (before the random transformation).

This process was applied to register the indoor point clouds. Then we used it to register the complete indoor point cloud with the outdoor point clouds.

\section{RESULTS}

Keypoints were rotated around a random axis with a random angle between 0 and pi/4. Then they were translated along a random axis with a random distance between zero and two meters. PCL's SIFTKeypoint and MATLAB's ICP had their input parameters tuned by experimenting different values. Mean error was measured for indoor registration over 350 point clouds.

Parameters for both algorithms are:

SIFTKeypoint:

Search method: kdtree

Minimum scale $=0.1$

Number of octaves $=6$

Number of scales per octave $=10$

Minimum contrast $=0.5$

ICP:

RMS difference $=1.0 \mathrm{e}-5$

Final overlap $=100 \%$
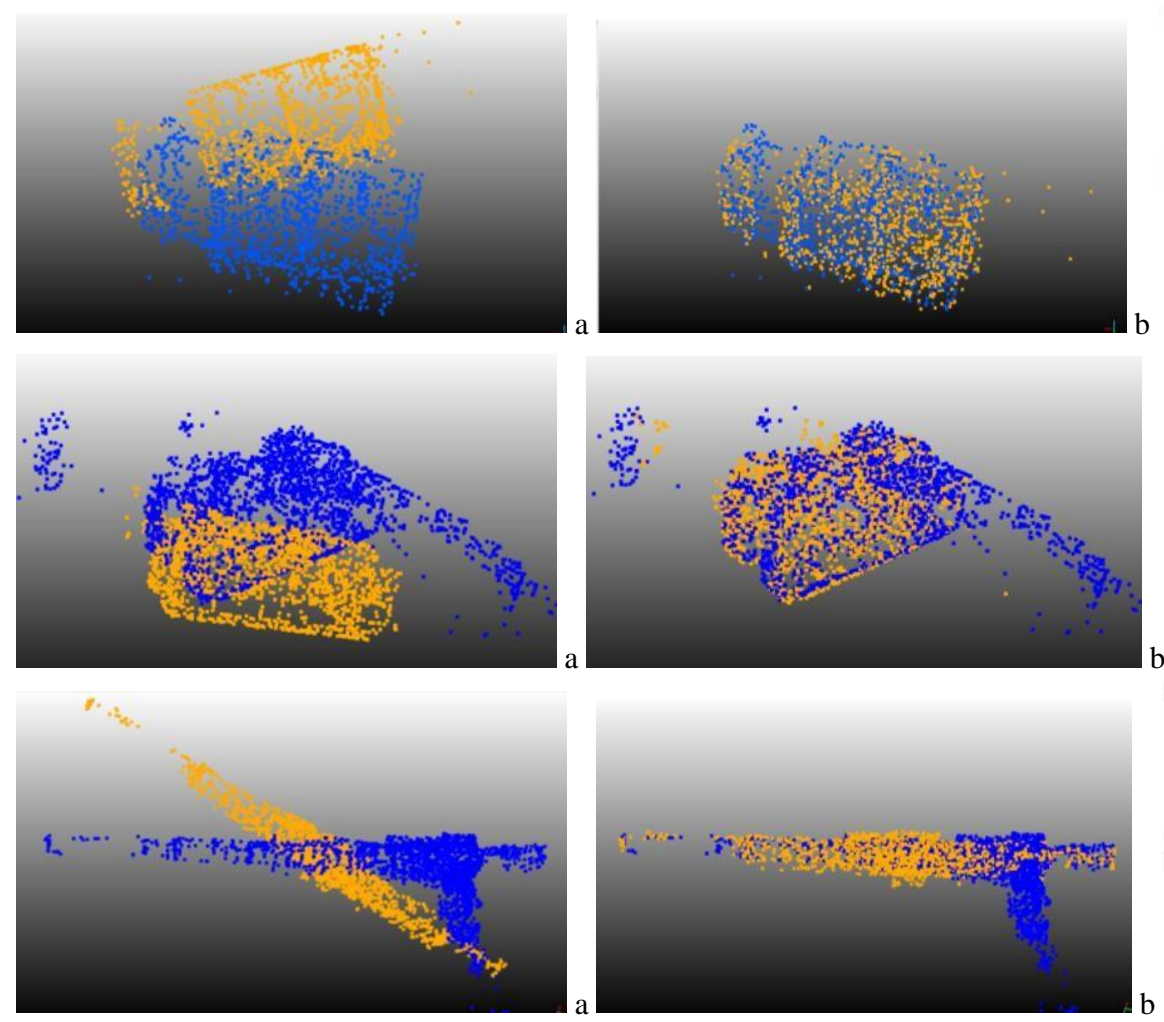

Figure 8. Examples of registration of 3 different pairs of indoor point clouds. a - The reference point cloud is blue, the randomly transformed data point cloud is orange. $\mathrm{b}$ - The data cloud is registered on the reference cloud with ICP. $\mathrm{c}-$ Histograms of the distances between registered points of the data cloud and their ground truth. Distances are in meters.
Gauss: mean $=0.170843 /$ std.dev. $=0.063715[50$ classes $]$

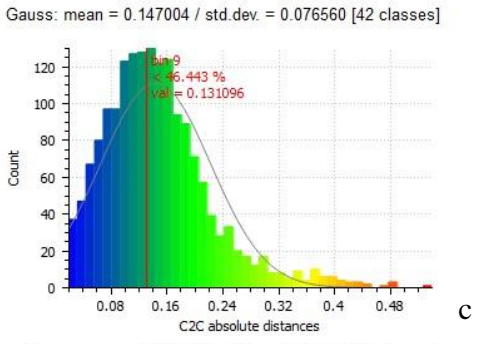

Gauss: mean $=0.141815 /$ std.dev. $=0.061630$ [53 classes $]$

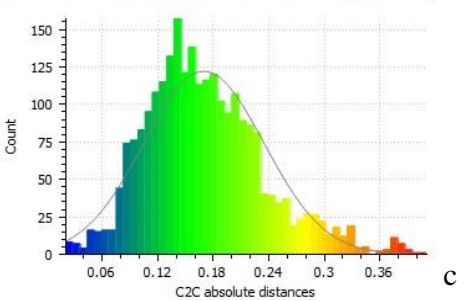

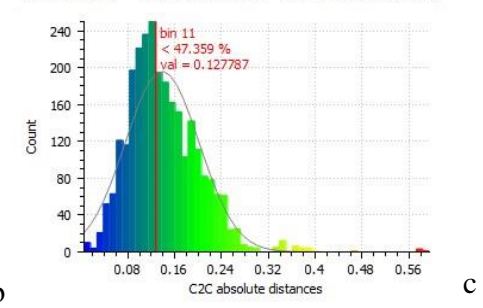




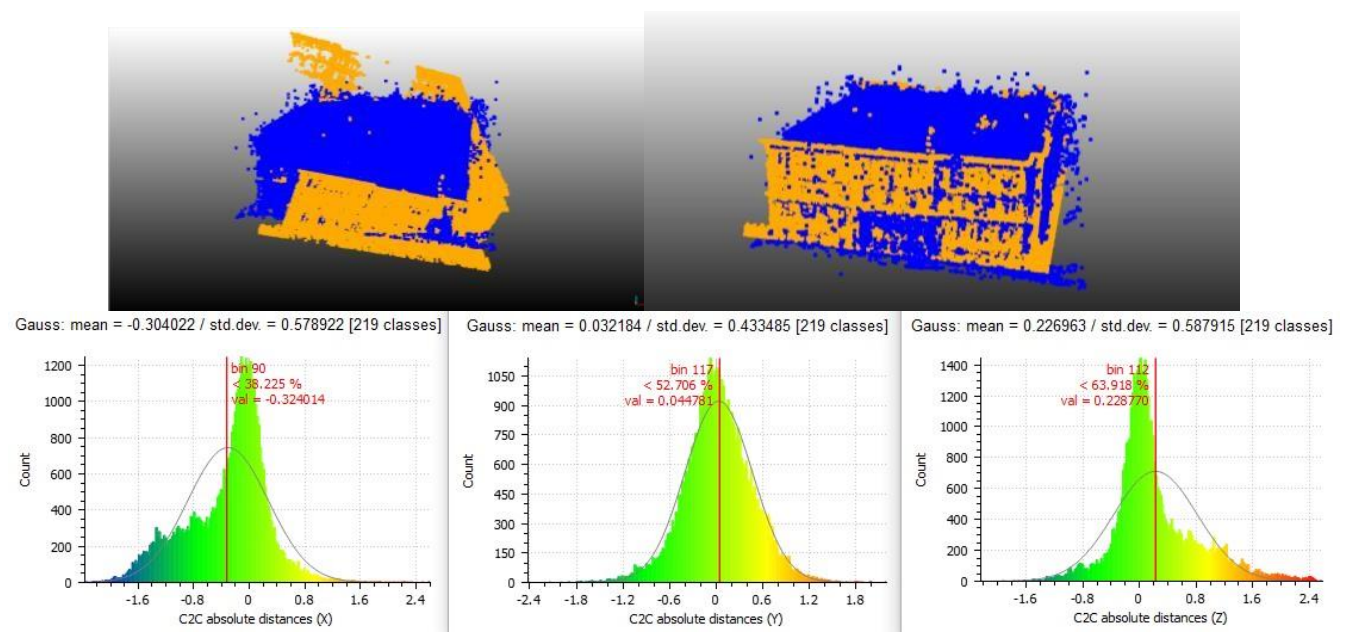

Figure 9. Joint indoor/outdoor point cloud registration. After a random transformation the keypoints of the orange façade were registered with ICP on the indoor point cloud (in blue). The histograms show the distances between registered points and their ground truth in meters along all three axis.

As we can see in Figure 8 and 9, the random transformation that we applied to "unregister" cannot be neglected. We wanted the data to appear as if it were manually and roughly registered. But the initial positions are actually much worse than what an operator could achieve. Yet the ICP was very efficient. It can be inferred that it would be equally or more efficient on initially roughly registered point clouds. The mean distances of registered keypoints to their ground truth is less than $20 \mathrm{~cm}$ (Figure 8). On average for our dataset, keypoints represent $3.24 \%$ of the original point cloud. This makes the registration step or any step following keypoints detection much faster.

Another interesting result is that registration using $100 \%$ of the point clouds give very poor result with ICP. In other words, registration is more efficient with only keypoints than with all points.

For joint indoor outdoor segmentation, we registered the point clouds of 3 façades on the indoor point cloud (Figure 9).

If we compare histograms of figures 8 and 9 , the indoor/outdoor registration is visually coherent and the mean error has similar value as the previous experiment. But a large number of points is badly registered with an error superior to one meter. Yet those results are still much better than registering complete RGB raw point clouds with ICP.

\section{CONCLUSION AND FUTURE WORKS}

In this study, we assessed the pertinence of using keypoints detected by SIFTKeypoint rather than the entirety of a point cloud for further processing. The experiments consisted in performing indoor/indoor point cloud registrations and indoor/outdoor point cloud registrations with ICP.

Applying only ICP for the registration of raw indoor and outdoor point clouds is impossible. However, when processing the keypoints with ICP, promising results were achieved. Indeed, mean error is inferior to $20 \mathrm{~cm}$ for indoor point clouds. And a slightly higher $80 \mathrm{~cm}$ for outdoor point cloud. The processing was also faster due to the smaller density of the keypoints: $3.2 \%$ of original point clouds.
This result was achieve with the most basic ICP. For future work, we will improve the global registration by looping over all available clouds rather than considering only two consecutive point clouds. This will let point clouds register with clouds that have the best registration score rather than prioritizing the order of the files.

To register parts of the façade with parts of the indoor cloud rather than the entire indoor cloud may also give better results. For instance if we can isolate only rooms close to the façade, the registration will not take useless indoor points into account. In order to refine the registration, more complex algorithms than the ICP should be tested. Adding features to the keypoints detection such as RGB information could also improve the results.

\section{REFERENCES}

Assi, R., Landes, T., Macher, H., Grussenmeyer, P., 2019. Energy Function Algorithm for Detection of Openings in Indoor Points Clouds, Int. Arch. Photogramm. Remote Sens. Spatial Inf. Sci., XLII-2/W13, 747-752, 2019.

Boulaassal, H., Landes, T., Chevrier, C., 2010. From Laser Data to Parametric Models: Towards an Automatic Method for Building Façade Modelling. EuroMed: Digital Heritage, pp. 4255 .

Boykov, Y., Veksler, O., Zabih, R., 2001. Fast approximate energy minimization via graph cuts. IEEE Transactions on pattern analysis and machine intelligence, volume 23 , issue $11, \mathrm{p}$ 1222-1239.

Lowe, D., 2004. Distinctive image features from scale-invariant keypoints. International Journal of Computer Vision, 60, 2, pp. 91-110.

Fischler, M. A., Bolles, R. C., 1981. Random Sample Consensus: A paradigm for model fitting with. In communications of the ACM, volume 24, pp. 381-395.

Macher, H., Landes, T., Grussenmeyer, P., 2017. From Point Clouds to Building Information Models: 3D Semi-Automatic 
Reconstruction of Indoors of Existing Buildings. Applied sciences 7(10), 1030, 30 pages. doi:10.3390/app7101030

Mittet, M-A., Landes, T., Grussenmeyer, P., 2014. Localisation using RGB-D cameras orthoimages, The International Archives of the Photogrammetry, Remote Sensing and Spatial Information Sciences, Volume XL-5, p 425-432.

Murtiyoso, A., Grussenmeyer, P., 2018. Comparison and assessment of 3D registration and georeferencing approaches of point clouds in the as exterior and interior heritage building. ISPRS TC II Mid-term Symposium "Towards Photogrammetry 2020”, 4-7, Riva del Garda, Italy ISPRS Archives p 744-751.

Rusu, D., Laguela, S., Diaz-Vilarino, L., Armesto, J., Arias, P., 2011. 3D is here: point cloud library. Proceedings of IEEE International Conference on Robotics on Automation. IEEE, Shanghai, pp. 1-4.

Torr, P.H., Zisserman, A., 2000. Mlesac: a new robust estimator with application to estimating image geomerty. Computer vision and image understanding, 78(1):138-156.

Wenzhong, S., Wael., A., Na, L., Wenzheng, F., Haodong, X., Muyang, W. 2019. Semantic Geometric Modelling of Unstructured Indoor Point Cloud. ISPRS Int. J. Geo-Inf. 8, 9.

20 pages; doi:10.3390/ijgi8010009 